\title{
Comparing online and professional restaurant reviews: What can we learn?
}

\author{
Ziye Zhang
}

Ziye is an emerging researcher in the field of hospitality management. She received her bachelor's degree from Beijing Union University in 2015 and her master's degree from Auckland University of

Technology in 2017, majoring in international hospitality management. Her research focusses on user-generated content in restaurant reviews, a relatively new data source for analysis in this area. Ziye also researches electronic word-of-mouth, online restaurant reviews and writers' criteria for restaurant measurement.

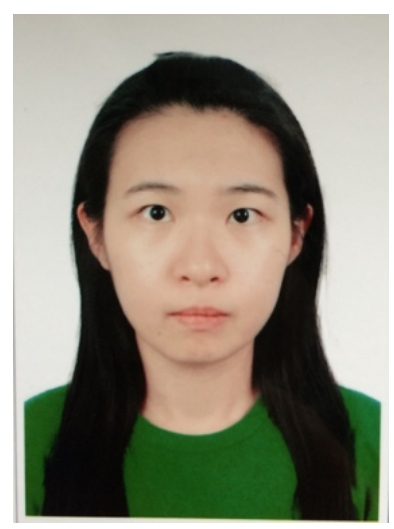

Social media and review websites such as Trip Advisor are emerging as important platforms for restaurant reviews. The tendency of consumers to seek out electronic word of mouth before deciding where to dine has increased rapidly, with online content being shown to significantly impact purchasing decisions. Practitioners have indicated they are keenly aware of the importance of online reviews, but there is almost no research that looks at this phenomenon in the New Zealand context, and no research that compares traditional, print-based reviews with online content. In order to address this gap, a recent Auckland University of Technology master's thesis applied content analysis to compare online and published restaurant reviews of restaurants in Auckland, New Zealand. Three hundred reviews from TripAdvisor website, Cuisine Magazine and the New Zealand Herald newspaper were analysed, and some results are presented here.

Traditional print reviews (Cuisine and the Herald) tend towards a more 'specialist' approach, including detailed information about the chefs, the owners and the restaurants' histories. They also use more 'fancy' language in their food descriptions, including very detailed menu ingredients. TripAdvisor reviews, by contrast, tend towards non-specialist, 'plain language' reviews, with simple menu descriptor words and little attention on ingredients. When discussing price, print reviews take an 'objective' stance, listing the price but rarely making a personal judgement about value; whereas the online reviews feature prominent personal judgements about value for money. In addition, online reviews emphasise the social aspects of dining (who they are dining with, the social nature of the event, e.g. birthday, interactions throughout the meal), often spending as much time discussing their companions as the products and service involved in the experience. By contrast, print reviews tend to focus more on the 'physical' aspects of dining (the wine, the food, the decor). Finally, both platforms strongly emphasise food and service as the main criteria for judgement, accounting for almost half the word counts in both styles of reviews.

What can the practitioner take from this research? Firstly, the fact that food and service are the predominant criteria for both online and professional reviewers serves as a reminder for restaurant operators that, no matter how exquisite or stylish the restaurant is, well-cooked food and attentive service are significantly emphasised as the primary criteria by reviewers. Moreover, both review formats stress that the reliability of the booking system and efficiency of the service staff tended to enhance all reviewers' satisfaction. 
However, there are clear differences in the review styles that represent the different audiences they are addressing. Each format attempts to recognise the perceived social identity of their audience, with the print reviews taking the 'insider expert' voice and appearing to speak to a more sophisticated upmarket, product-focussed audience. The print reviews also assume their readers are less sensitive to price, rarely discussing the perceived value of the meal. By contrast, the online reviews are more 'plain language', discuss value directly and are socially driven. By being aware of these differences in reviewing approaches, restaurant operators' can finesse their marketing strategies. By reading and reflecting on the link between the contrasting review styles and the social identity of the respective readers, practitioners can consider how their offering 'fits' with their target markets. Finally, as online review platforms become increasingly important, practitioners should engage with the content they find there, ensuring they take time for reading, reflecting and responding.

If you would like to read the original thesis this article is based on, please email: david.williamson@aut.ac.nz

\section{Corresponding author}

Ziye Zhang can be contacted at: ziye0927@outlook.com 